\title{
Promoting the use of Personal Asthma Action Plans: a systematic review
}

\section{* Nicola Ringa, Cari Malcolma, Sally Wykea, Steve MacGillivrayb, Diane Dixonc, Gaylor Hoskins ${ }^{d}$, Hilary Pinnocke, Aziz Sheikhe}

\author{
alliance for Self-Care Research, Department of Nursing \& Midwifery, University of Stirling, Stirling, FK9 4LA, Scotland, UK \\ ${ }^{\text {b }}$ Alliance for Self-Care Research, Tayside Institute for Health Studies, University of Abertay, Dundee, Scotland, UK \\ ' Department of Psychology, University of Stirling, Stirling, Scotland, UK \\ d Tayside Centre for General Practice, Community Health Sciences, University of Dundee, Scotland, UK \\ e Allergy \& Respiratory Research Group, Division of Community Health Sciences:GP Section, University of Edinburgh, Scotland, UK
}

Received 27th February 2007; accepted 15th May 2007

\begin{abstract}
Objectives: To investigate how best to encourage health professionals to promote, and for people with asthma to use, asthma action plans.

Methods: Systematic review. Randomised controlled trials published between 1960 and 2006 were searched using multiple electronic databases. Unpublished and ongoing studies were identified by contacting asthma experts internationaily. Included trials reported outcome data for the promotion of action plans including issue of plans by health professionals, and patient ownership and use.

Results: 14 trials satisfied our study inclusion criteria. Of these, only four studies reported data for action plan use. Interventions included: education of doctors and people with asthma; telephone reinforcement; partially completed action plans and postal prompts inviting patients for general practice review; school asthma clinics; and asthma management systems (including the 3+ plan with patient recall for review and Internet-based physician monitoring). These interventions increased action plan ownership, use, or facilitation of use. Two of the highest quality papers were conducted in primary care and demonstrate the effectiveness of interventions directed at the organisation of asthma care in promoting action plan use.

Conclusions: Primary care professionals could encourage the ownership and use of action plans through the implementation of proactive practice-based organisational systems, though further research is required to assess their practicality and effect on sustaining use long-term. Multi-disciplinary teams working in areas where asthma action plan ownership and use is sub-optimal should therefore consider how such interventions could be incorporated into existing practices and healthcare systems.
\end{abstract}

(C) 2007 General Practice Airways Group. All rights reserved.

N Ring, et al. Prim Care Resp J 2007; 16(5): 271-283.

doi:10.3132/pcrj.2007.00049

Keyw ords asthma, asthma action plans, use, health education, self-management, interventions, systematic review

\section{Introduction}

Written personal asthma action plans within a selfmanagement programme are known to improve clinical outcomes for people with asthma, ${ }^{1}$ are recommended as good practice in asthma care, ${ }^{2-4}$ and there is clear guidance about their component parts. ${ }^{5}$ Their use by people with asthma also facilitates self-management, which is currently being encouraged in chronic disease management internationally. ${ }^{6-10}$

If people with asthma are to use action plans they need to be issued with them, understand their role, and know how to use them appropriately. There is, however, evidence that they are under-provided by health professionals, under-utilised by those with asthma, ${ }^{11-15}$ and not always updated following treatment changes. ${ }^{16}$ This paper reports the findings of a systematic review commissioned by Asthma UK (Scotland) and NHS Quality Improvement Scotland to investigate how best to encourage health professionals to promote, and for people with asthma to use, asthma action plans.

\footnotetext{
* Corresponding author: Tel: +44 (0)1786 466351 Fax: +44 (0)1786 466333 E-mail: nicola.ring@stir.ac.uk
} 
Figure 1. Flow chart detailing the published literature screening process.

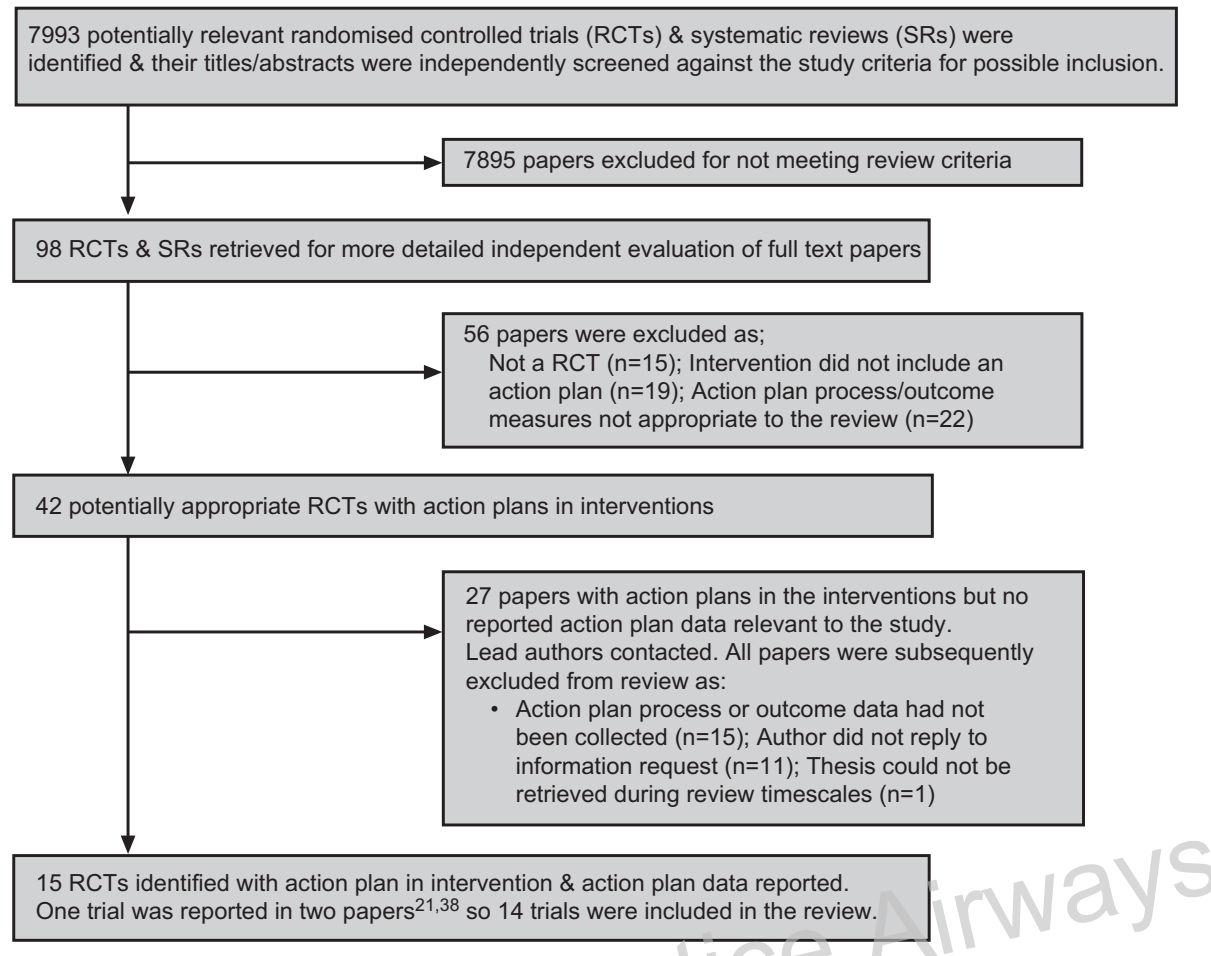

1. The first and second authors (NR \& CM) independently screened study titles, abstracts and full papers and referred any disagreements to the wider team for arbitration. NR and CM also independently extracted data from the 14 included studies. The full team reviewed all included studies and completed data extraction forms for completeness and accuracy. Literature screening was conducted in 2006.

2. The above is based on the QUORUM flowchart ${ }^{39}$

\section{Methods}

\section{Definition}

Action plans were defined as written or electronic records agreed by a person with asthma (or their parent/carer) and their health professional(s) providing personalised guidance on what action to take when asthma symptoms or peak flow deteriorate. ${ }^{17}$

\section{Types of studies}

Only randomised controlled trials (RCTs) - or systematic reviews of RCTs - assessing the effectiveness of an intervention encouraging health professionals (or others) to promote action plan use amongst patients and/or encourage those with asthma to use action plans were included. Studies had to report quantifiable action plan data such as increased distribution by health professionals and/or increased use by people with asthma; other asthma outcomes including use of medication and health services were outwith the remit of this review. Studies not meeting these criteria were excluded.

\section{Study identification}

Multiple electronic databases were searched from 1960 to 2006 (MEDLINE, EMBASE, CINAHL, PsycINFO, ERIC, Cochrane Library, DaRE, SSCI and SCI). A combination of medical subject headings (MeSH) and keywords were used. Key search terms were mapped to existing subject headings, and included asthma, respiratory disease, action plans, selfmanagement plans and trials. Searches were not limited by language or year, and resulted in 7,993 potential papers.

Figure 1 details the published literature screening process. The first two authors independently screened all titles and abstracts, identifying 98 possible studies. These full papers were then independently reviewed. Fifty-six papers did not meet our inclusion criteria, leaving 42 possible RCTs. Twenty-seven of these included an action plan within their intervention but did not report any data on action plan ownership or use, so lead authors were contacted for additional information. As relevant data had either not been collected or lead authors did not reply, these studies were subsequently excluded. Fourteen studies were identified for inclusion in the review and their reference lists were screened, but no additional trials were identified.

To locate unpublished and/or on-going studies, asthma experts internationally were identified via searches of academic publications and the Internet, and were contacted for relevant information. Although 26 authors and three groups replied (of the 74 authors and 29 groups contacted), no further studies were found which met the inclusion criteria. 


\section{Results}

\section{Study characteristics}

The 14 included studies were all RCTs, ${ }^{18-31}$ although Salisbury ${ }^{26}$ also included a parallel observation arm. Data from the 14 trials were systematically extracted onto a specially designed form. Summaries of the 14 trials including type of action plans, interventions, and key findings (but excluding participant details) are shown in Table 1.

Studies were published between 1993-2005 in the UK, ${ }^{24-27}$ Australia, ${ }_{18,20,22,28,29}$ United States, ${ }^{21,30}$ Denmark, ${ }^{31}$ Canada, ${ }^{23}$ and New Zealand. ${ }^{19}$ The methodological quality of these RCTs was assessed for performance, detection and attrition bias, and was graded ' $A$ ' (low risk of bias) to ' $C$ ' (high risk). ${ }^{32}$ One study was graded ' $A$ ', ${ }^{28}$ three were graded ' $B$ ', ${ }^{25,27,30}$ and the remainder were graded ' $C$ '. . $^{8-24,26,29,31}$

Participants were people with asthma (or their parents) $(n=4438)$, doctors $(n=107)$ and general practice teams $(n=43)$ (Table 2). Studies investigated either adults (over 16 years) only, ${ }_{1}^{18,22-24,27,31}$ adults and children, ${ }^{19,20,25,29}$ children only, ${ }_{1}^{26}$ or children with their health professionals. ${ }^{21,28,30}$ Most participants had moderate-to-severe asthma ${ }^{22,28}$ or were recruited following medical care for acute asthma. ${ }^{18,19,21,23-25,29}$ Studies used various recruitment strategies (Table 2) including general practice registration/attendance, ${ }^{20,27}$ hospital admission, ${ }^{24,25}$ and questionnaire completion. ${ }^{26,28,31}$ Two studies included patients over 70 years of age. ${ }^{24,27}$ Six studies provided participant demographic details including ethinicity and deprivation. $18,19,21,23,25,30$

\section{Complexity of interventions, variability in setting, and outcome reporting}

Action plans were usually included within a complex intervention ${ }^{33}$ - for example, self-management education - so it was not possible to determine which component of an intervention impacted on action plan use. Interventions were varied, reflecting the many contexts in which action plan use needs to be encouraged. Studies also reported a wide range of action plan measures, sometimes using only descriptive statistics and/or with occasional inconsistencies in statistical data reporting. Such diversity of interventions and reporting meant that it was not appropriate to synthesise data quantitatively; therefore, a narrative approach was adopted.

\section{Outcomes}

For this review, the primary outcomes of interest were action plan-specific such as people with asthma having and using these plans. Amongst the trials which specified their primary outcomes, only two cited action plan measures, ${ }^{27,30}$ with the remainder citing clinical measures - including medication use and hospitalisation rates - as their primary focus. ${ }^{22,23,25,26,29}$

The language used to describe similar action plan outcomes varied considerably, and some inconsistencies were noted (see Table 1). For example, in one paper the abstract cited action plan use as an outcome, the text referred to patients receiving action plans, whilst tabular data reported patients having action plans..$^{20}$ Generally, outcome measures such as patients having or using action plans were not defined in the original trials (Table 1). Consequently, it was extremely difficult to construct a comprehensive narrative of our findings.

To provide a consistent framework from which to understand our results, we therefore grouped reported action plan outcomes according to ownership and use. 'Ownership' included patients/parents with action plans, and professionals distributing plans to patients. 'Use' included frequency of action plan use by patients/parents and how professionals facilitated their use (for example, by increasing patient understanding of how to use action plans).

Measures relating to the delivery of care were most frequently reported, especially patients receiving action plans. ${ }^{18-20,24-26,28-30}$ Outcome measures such as action plan use and understanding were reported less often. ${ }^{22,23,27,29,31}$ Analysis of action plan data by participant sub-groups was also generally absent, making sub-group analysis within this systematic review non-feasible. For example, only four studies $^{19,27-29}$ reported age-specific data. Only Clark ${ }^{21}$ reported data for more than one year post-intervention.

\section{Interventions}

Most interventions $(n=11)$ were conducted in a non-hospital setting using health professionals; two studies ${ }^{19,22}$ used nonhealthcare workers. Generally, scant information was provided about the action plans used (see Table 1). Where action plans were said to have been individualised, no details were provided about how this had been achieved. The design of some interventions excluded participation from certain groups. For example, some studies required participants to have telephone or computer access ${ }^{22,29,31}$ or a level of English literacy. ${ }^{19}$

Interventions could be broadly described as being aimed at people with asthma (or their parents) 18-20,22-29,31 or their health professionals ${ }^{21,30}$ (see Table 1). Of the two studies which focused primarily on professionals, ${ }^{21,30}$ only one used a multidisciplinary intervention aimed at a primary care team. ${ }^{30}$

Overall, interventions could be categorised as educational, 18,19,21,23-25 reinforcing or prompting, ${ }^{22,27,29}$ asthma clinics, ${ }^{20,26}$ asthma management systems, ${ }^{28,31}$ and quality improvement. ${ }^{30}$ All interventions except one ${ }^{30}$ had some effect on action plan process or outcome measures.

\section{Interventions aimed at people with asthma}

Self-management education aimed at people with asthma was the most frequent intervention. 18,19,23-25 Four such trials showed a significant increase in the number of people with asthma (or their parents) with action plans in the intervention compared to control group ${ }^{18,19,24,25}$ (see Table 1). One other study ${ }^{23}$ reported significantly higher action plan use following structured group or individual self-management education 
Table 1. Characteristics of included studies (excluding participant details).

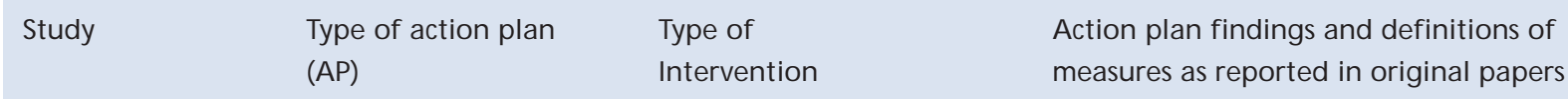

\begin{tabular}{|c|c|c|c|}
\hline \multicolumn{4}{|c|}{ A) Interventions aimed at people with asthma } \\
\hline $\begin{array}{l}\text { Adams }^{22} \text { (2001) } \\
\text { RCT } \\
\text { Quality grading C } \\
\text { Duration: } 12 \text { months. }\end{array}$ & $\begin{array}{l}\text { Peak flow (PF) or symptom } \\
\text { monitoring (SM). Paper } \\
\text { refers to AP and self- } \\
\text { management plans. } \\
\text { Copy of plan in paper. Plan } \\
\text { based on Australian } \\
\text { National Asthma } \\
\text { Campaign } \\
\text { recommendations. }\end{array}$ & $\begin{array}{l}\text { Monthly telephone calls by a } \\
\text { non health care worker to } \\
\text { patients encouraging AP use } \\
\text { over a one year period. (AP } \\
\text { given to patients by doctor). }\end{array}$ & $\begin{array}{l}\text { Most participants used their AP appropriately: PF group } \\
86 \% \text { ( } n=57 \text { ) \& SM group } 85 \% \text { ( } n=50 \text { ). } \\
\text { At } 12 \text { months, } 76 \% \text { ( } n=56 \text { ) of the PF group \& } 78 \% \text { ( } n=47 \text { ) } \\
\text { of the SM group either had/gained an understanding about } \\
\text { using an AP to manage their asthma. } \\
8 \% \text { ( } n=6 \text { ) of the PF group had symptoms before their peak } \\
\text { expiratory flow readings dropped enough to trigger use of } \\
\text { the AP. } \\
5 \% \text { ( } n=6 \text { ) of the SM group either had different symptoms to } \\
\text { those on their AP or had such rapid onset of symptoms that } \\
\text { the plan could not be initiated. } \\
10 \% \text { ( } n=13 \text { ) would only change their medication after } \\
\text { medical approval. } \\
9 \% \text { ( } n=12 \text { ) of participants never understood the concept of } \\
\text { an AP. } \\
10 \% \text { ( } n=14 \text { ) chose to be non-compliant with their AP even } \\
\text { if they knew how to use it. } \\
\text { Inappropriate use defined as, for example, patient not } \\
\text { changing medication as per plan. AP compliance was } \\
\text { assessed by study co-ordinator and included patient's ability } \\
\text { to adhere to plan and understanding of use. }\end{array}$ \\
\hline $\begin{array}{l}\text { Cote }^{23}(2001) \\
\text { RCT } \\
\text { Quality grading C } \\
\text { Duration: } 12 \text { months. }\end{array}$ & $\begin{array}{l}\text { PF or symptom based. } \\
\text { Plans used traffic light } \\
\text { system. Details in text }\end{array}$ & $\begin{array}{l}\text { Limited \& structured } \\
\text { community education } \\
\text { programme (group or one-to- } \\
\text { one teaching) based on } \\
\text { PRECEDE model. Two } \\
\text { educational contacts. 1st } \\
\text { following acute asthma } \\
\text { exacerbation. } 2 \text { nd } 6 \text { months } \\
\text { later to provide reinforcement. } \\
\text { Education provider unknown } \\
\text { but conducted in a } \\
\text { community education centre. }\end{array}$ & $\begin{array}{l}\text { At six months the proportion of participants using APs was } \\
\text { comparable between the limited education (LE) \& structured } \\
\text { education (SE) groups ( } p=0.18 \text { ) (approximately } 22 \% \& 40 \% \\
\text { respectively**). } \\
\text { At one year post intervention, the SE group had a } \\
\text { significantly higher AP use ( } p=0.008 \text { ) than the LE group } \\
\text { (approximately } 68 \% \& 18 \% \text { respectively **). } \\
{ }^{* *} \text { data were presented in a bar chart; however, it was } \\
\text { difficult to interpret exact percentages from the paper). } \\
\text { AP use or implementation not defined in paper. }\end{array}$ \\
\hline $\begin{array}{l}\text { Garrett }^{19} \text { (1994) } \\
\text { RCT } \\
\text { Quality grading C } \\
\text { Duration: } 9 \text { months. }\end{array}$ & $\begin{array}{l}\text { Individualised written plan } \\
\text { advising patients on what } \\
\text { to do if asthma worsened. }\end{array}$ & $\begin{array}{l}\text { Community education } \\
\text { programme by a nurse } \\
\text { specialist \& trained non } \\
\text { healthcare worker. Frequency } \\
\& \text { duration of education not } \\
\text { specified. Group or one-to- } \\
\text { one teaching in a community } \\
\text { education centre. }\end{array}$ & $\begin{array}{l}\text { Both groups reported higher numbers with AP at follow up } \\
\text { but this was greatest amongst children }(p=0.0001) \text { \& adults } \\
(p=0.01) \text { who had attended a community education centre } \\
\text { staffed by a nurse \& non health care community workers. } \\
\text { Pre- and post-education } 22 \%(n=50) \& 61 \%(n=139) \text { of } \\
\text { intervention participants had APs, but these data are not } \\
\text { presented separately for children \& adults. } \\
\text { Significant improvement in knowledge regarding actions to } \\
\text { take during an asthma exacerbation also increased more in } \\
\text { adults ( } p=0.005) \text { who received the education. } \\
\text { Definitions of AP measures not provided in paper. }\end{array}$ \\
\hline
\end{tabular}


Table 1. Continued.

\begin{tabular}{|c|c|c|c|}
\hline Study & $\begin{array}{l}\text { Type of action plan } \\
\text { (AP) }\end{array}$ & $\begin{array}{l}\text { Type of } \\
\text { Intervention }\end{array}$ & $\begin{array}{l}\text { Action plan findings and definitions of } \\
\text { measures as reported in original papers }\end{array}$ \\
\hline $\begin{array}{l}\text { Glasgow }^{28}(2003) \\
\text { RCT } \\
\text { Quality grading A } \\
\text { Duration: } 12 \text { months. }\end{array}$ & $\begin{array}{l}\text { Written asthma plan - no } \\
\text { details given. }\end{array}$ & $\begin{array}{l}\text { 3+ plan (proactive asthma } \\
\text { management plan) combined } \\
\text { with active recall system } \\
\text { inviting children to attend for } \\
\text { asthma review in primary care. }\end{array}$ & $\begin{array}{l}\text { Using the } 3+\text { asthma management plan more children had } \\
\text { an AP at } 12 \text { months compared to those with usual GP care } \\
\text { ( } 44 \% \text { versus } 34 \% \text {; odds ratio } 2.295 \% \mathrm{Cl} 1.2-4.1)(p=0.01 \text { ). } \\
\text { NNT: } 5 \text { extra children would need to receive the } 3+\text { plan \& } \\
\text { active recall system for one extra AP to be given ( } 95 \% \mathrm{Cl} 3- \\
\text { 41). } \\
\text { The term having an AP was not defined in the paper. }\end{array}$ \\
\hline $\begin{array}{l}\text { Heard }^{20} \text { (1999) } \\
\text { RCT } \\
\text { Quality grading C } \\
\text { Duration: } 6 \text { months }\end{array}$ & $\begin{array}{l}\text { Written asthma } \\
\text { management plan - no } \\
\text { details given. }\end{array}$ & $\begin{array}{l}\text { Attendance at a general } \\
\text { practice asthma clinic using } \\
\text { self management education. } \\
\text { Asthma educator (a nurse } \\
\text { with extensive respiratory } \\
\text { experience) \& GP at clinic. }\end{array}$ & $\begin{array}{l}\text { At six months follow-up, } 75 \% \text { of patients who were } \\
\text { randomised to attendance at a primary care based asthma } \\
\text { clinic were in receipt of a written AP compared to } 65 \% \text { of } \\
\text { patients who attended for standard care. When adjusted for } \\
\text { baseline measures \& clustered by doctor, this represents an } \\
\text { odds ratio of } 1.62 \text { ( } 95 \% \mathrm{Cl} 0.82-3.22 \text { ). } \\
\text { The issuing of AP increased significantly in both groups } \\
\text { (intervention group: odds ratio } 11.25 ; 95 \% \mathrm{Cl} 3.07-41.21 \text { \& } \\
\text { control group: odds ratio } 3.94,95 \% \mathrm{Cl} 1.53-10.10) \text {. } \\
\text { AP measures such as receiving or having were not defined } \\
\text { in paper. }\end{array}$ \\
\hline $\begin{array}{l}\text { Kemple }^{27} \text { (2003) } \\
\text { RCT } \\
\text { Quality grading B } \\
\text { Duration: } 12 \text { months }\end{array}$ & $\begin{array}{l}\text { Written AP - no details } \\
\text { given. }\end{array}$ & $\begin{array}{l}\text { Postal prompts inviting } \\
\text { patients with asthma to } \\
\text { attend for review plus a } \\
\text { partially completed } \\
\text { personalised AP (Group 3) or } \\
\text { a blank AP (Group 2) for } \\
\text { completion at the review. } \\
\text { Participants receiving usual } \\
\text { care were Group 1. }\end{array}$ & 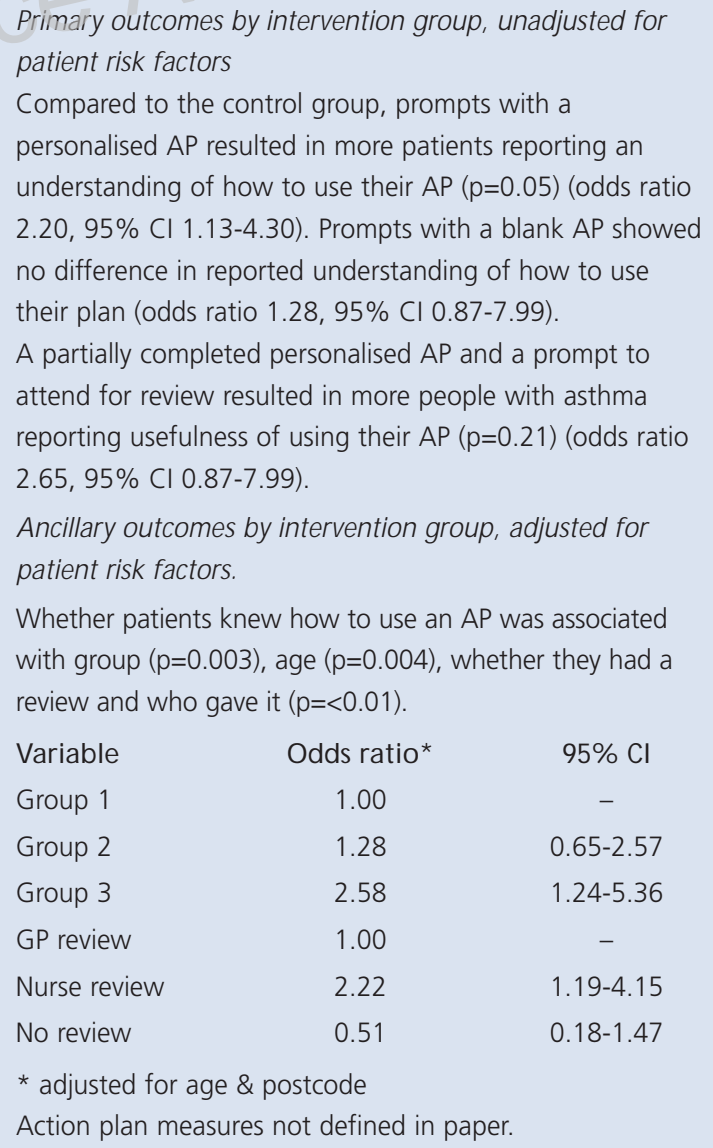 \\
\hline
\end{tabular}


$\mathrm{N}$ Ring et al.

Table 1. Continued.

\begin{tabular}{|c|c|c|c|}
\hline Study & $\begin{array}{l}\text { Type of action plan } \\
\text { (AP) }\end{array}$ & $\begin{array}{l}\text { Type of } \\
\text { Intervention }\end{array}$ & $\begin{array}{l}\text { Action plan findings and definitions of } \\
\text { measures as reported in original papers }\end{array}$ \\
\hline $\begin{array}{l}\text { Khan }{ }^{29}(2004) \\
\text { RCT } \\
\text { Quality grading C } \\
\text { Duration: } 6 \text { months. }\end{array}$ & $\begin{array}{l}\text { Personalised written AP - } \\
\text { no details. }\end{array}$ & $\begin{array}{l}\text { One-off telephone } \\
\text { consultation (lasting } 13 \\
\text { minutes on average, } \\
\text { range 5-44 minutes) } \\
\text { from a trained asthma } \\
\text { educator (nurses) to } \\
\text { parents of children with } \\
\text { asthma following } \\
\text { accident \& emergency } \\
\text { attendance. }\end{array}$ & $\begin{array}{l}\text { Significantly more participants in the intervention group had } \\
\text { an AP at } 6 \text { months follow-up compared to control group } \\
\text { participants ( } 88 \% \text { ( } n=119 \text { ) versus } 72 \% \text { ( } n=94 \text { ); } p=0.001 \text { ). } \\
\text { Frequency of use was also greater in the intervention group } \\
\text { with } 32 \% \text { ( } n=43 \text { ) using their AP often (compared to } 22 \% \text {, } \\
n=28 \text { of controls) \& } 56 \% \text { ( } n=76 \text { ) using their AP occasionally } \\
\text { (compared to } 51 \%, n=66 \text { for controls). } \\
\text { NNT: } 6.5 \text { children would need education \& monthly } \\
\text { telephone reinforcement for one additional child to get an } \\
\text { AP. } \\
\text { Study outcomes described as possession and use of an AP } \\
\text { during a crisis. Frequency of use - that is, whether APs were } \\
\text { used often or occasionally - was not defined and was } \\
\text { measured based on parental questionnaire response. }\end{array}$ \\
\hline $\begin{array}{l}\text { Morice }^{24} \text { (2001) } \\
\text { RCT } \\
\text { Quality grading C } \\
\text { Duration: } 6 \text { months. }\end{array}$ & $\begin{array}{l}\text { Written management plan } \\
\text { using Sheffield Asthma } \\
\text { Card [24]. }\end{array}$ & $\begin{array}{l}\text { Two individual patient } \\
\text { education sessions (each } \\
\text { lasting about } 30 \text { minutes) } \\
\text { prior to hospital discharge by } \\
\text { specialist asthma nurse. }\end{array}$ & $\begin{array}{l}\text { At six weeks significantly more of the patients who had } \\
\text { received the education had an AP compared to those who } \\
\text { received usual care }(87 \% \text { compared to } 10 \% \text { respectively; } \\
p<0.001) \text {. } \\
\text { Significant differences in numbers with APs was also noted } \\
\text { at six months with } 86 \% \text { of the intervention group having an } \\
\text { AP compared to } 17 \% \text { of the control group ( } p<0.01) \text {. } \\
\text { AP measures not defined in text. }\end{array}$ \\
\hline $\begin{array}{l}\text { Osman }{ }^{25}(2002) \\
\text { RCT } \\
\text { Quality grading } B \\
\text { Duration: } 12 \text { months. }\end{array}$ & $\begin{array}{l}\text { Self management plan } \\
\text { used PF and symptoms. } \\
\text { Copy of AP in paper. }\end{array}$ & $\begin{array}{l}\text { Two individual patient } \\
\text { education sessions (each } \\
\text { lasting about } 30 \text { minutes) } \\
\text { prior to hospital discharge by } \\
\text { specialist asthma nurse. }\end{array}$ & $\begin{array}{l}93 \% \text { ( } n=98 \text { ) of the intervention group compared to } 30 \% \\
\text { ( } n=35 \text { ) of the control group had an AP }(p<0.0001) \text { at one } \\
\text { month post-intervention (odds ratio } 28.0,95 \% \mathrm{Cl} 12.3-63.8 \text {, } \\
p<0.0001 \text { ). } \\
\text { For patients subsequently requiring hospital admission: } \\
\text { At first re-admission: more patients in the intervention group } \\
\text { had an AP than control group patients ( } 98 \% \text { versus } 37 \% \text {, } \\
\text { odds ratio infinitely large, } p<0.0001 \text { ). } \\
\text { On multiple re-admission: more patients in the intervention } \\
\text { group had an AP than control group patients ( } 84 \% \text { versus } \\
25 \% \text {, odds ratio } 15.4,95 \% \mathrm{Cl} 6.0-39.7, p<0.0001) \text {. } \\
\text { AP measures not defined in paper. }\end{array}$ \\
\hline $\begin{array}{l}\text { Rasmussen }{ }^{31} \text { (2005) } \\
\text { RCT } \\
\text { Quality grading C } \\
\text { Duration: } 6 \text { months. }\end{array}$ & $\begin{array}{l}\text { AP with } 3 \text { colour warning } \\
\text { system \& written } \\
\text { treatment plan. Also, one } \\
\text { group got NHS Denmark } \\
\text { AP. }\end{array}$ & $\begin{array}{l}\text { Internet-based physician } \\
\text { asthma monitoring system or } \\
\text { out-patient based physician } \\
\text { support compared to GP } \\
\text { support. }\end{array}$ & $\begin{array}{l}\text { Significantly more patients using an Internet-based AP } \\
\text { reported using their AP at six month follow-up ( } 88 \%) \text { than } \\
\text { those who received an AP from a specialist via an out-patient } \\
\text { clinic }(66 \%) \text { or those that received usual care from a GP } \\
(6 \%)(p<0.001) \text {. } \\
\text { AP use not defined beyond asking patients to say yes/no to } \\
\text { whether they used their plan. }\end{array}$ \\
\hline
\end{tabular}




\section{Table 1. Continued.}

\begin{tabular}{|c|c|c|c|}
\hline Study & $\begin{array}{l}\text { Type of action plan } \\
\text { (AP) }\end{array}$ & $\begin{array}{l}\text { Type of } \\
\text { Intervention }\end{array}$ & $\begin{array}{l}\text { Action plan findings and definitions of } \\
\text { measures as reported in original papers }\end{array}$ \\
\hline $\begin{array}{l}\text { Salisbury }{ }^{26} \text { (2002) } \\
\text { RCT \& parallel } \\
\text { observation arm. } \\
\text { Quality grading C } \\
\text { Duration: } 6 \text { months. }\end{array}$ & $\begin{array}{l}\text { Written self management } \\
\text { plan - no details provided. }\end{array}$ & $\begin{array}{l}\text { Attendance (at least once) at } \\
\text { a school asthma clinic for } \\
\text { adolescents. Clinics run by a } \\
\text { school nurse with asthma } \\
\text { training. }\end{array}$ & 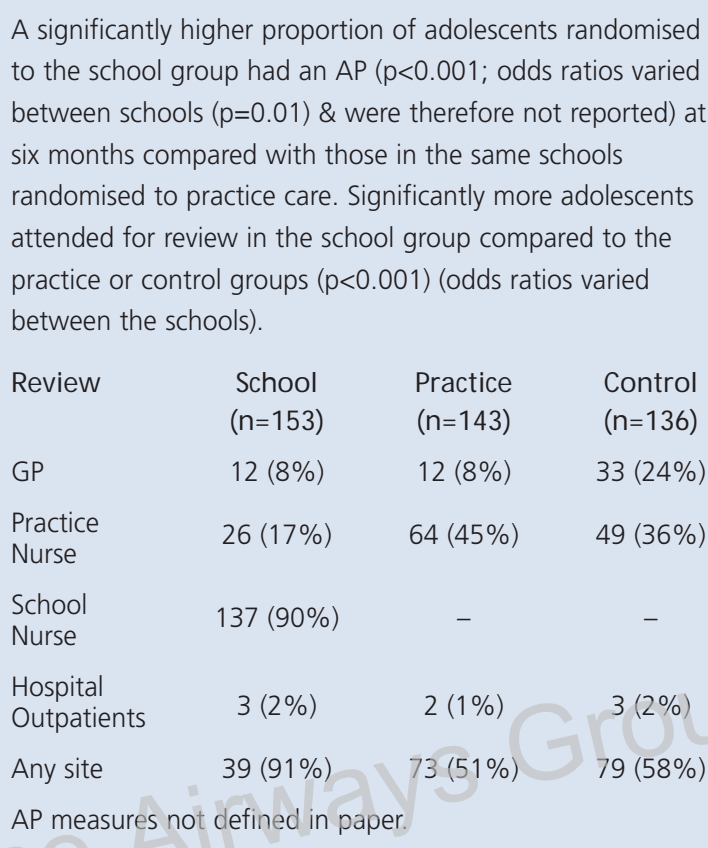 \\
\hline $\begin{array}{l}\text { Yoon }^{18} \text { (1993) } \\
\text { RCT } \\
\text { Quality grading } \\
\text { Duration: } 10 \mathrm{mo}\end{array}$ & $\begin{array}{l}\text { Treatment plan based on } \\
\text { peak flow - plan } \\
\text { referenced an earlier } \\
\text { paper [40]. }\end{array}$ & $\begin{array}{l}\text { Group self management } \\
\text { education (one session } \\
\text { lasting 2-3 hours) in a } \\
\text { community education centre. } \\
\text { Not known who provided } \\
\text { education. }\end{array}$ & $\begin{array}{l}\text { Intervention group had higher AP ownership at } 10 \text { month } \\
\text { follow up. } \\
\text { Mean (SD) scores of AP ownership at baseline and } 10 \\
\text { month follow up: } \\
\text { Baseline: Intervention } 2.89 \text { (1.61) Control } 3.32(1.46) \\
10 \text { months: Intervention } 4.79 \text { (1.57) Control } 3.03 \text { (1.91) } \\
\text { (F ratio 14.37, p=< 0.001) } \\
\text { AP ownership not defined in paper. }\end{array}$ \\
\hline
\end{tabular}

B) Interventions aimed at health professionals

\begin{tabular}{|c|c|c|c|}
\hline $\begin{array}{l}\text { Clark }^{21}(2000) \\
\text { (also reported in ref } \\
38) \\
\text { RCT } \\
\text { Quality grading C } \\
\text { Duration: } 2 \text { years. }\end{array}$ & $\begin{array}{l}\text { No details given. } \\
\text { Refers to treatment plan \& } \\
\text { therapeutic plan. }\end{array}$ & $\begin{array}{l}\text { Interactive educational } \\
\text { seminar for doctors based on } \\
\text { the theory of self-regulation. } \\
\text { Two face-to-face group } \\
\text { meetings over a three week } \\
\text { period. Not stated who } \\
\text { provided education or where. }\end{array}$ & $\begin{array}{l}\text { At two years more patients in the intervention group had } \\
\text { written information from the doctor informing them about } \\
\text { adjusting medications in response to symptom changes } \\
\text { ( } 29 \%, n=10 \text { compared to } 6 \% n=2 \text { for the control group; } \\
\text { odds ratio } 5.7, p=0.05 \text { ). } \\
\text { Having an AP not defined in paper. } \\
\text { Significantly more parents in the intervention group } \\
\text { reported the doctor commended them for taking the right } \\
\text { management action ( } p=0.02 \text { ), enquired about their fears \& } \\
\text { concerns associated with new medicines ( } p=0.02 \text { ), explained } \\
\text { the short term therapeutic plan describing what the family } \\
\text { needed to do before the next visit ( } p=0.03) \& \text { made it easier } \\
\text { for families to follow medication instructions ( } p<0.004 \text { ). }\end{array}$ \\
\hline
\end{tabular}




\begin{tabular}{l|l|l|l}
$\begin{array}{l}\text { Table 1. Continued. } \\
\text { Study }\end{array}$ & $\begin{array}{l}\text { Type of action plan } \\
\text { (AP) }\end{array}$ & \multicolumn{1}{l}{$\begin{array}{l}\text { Type of } \\
\text { Intervention }\end{array}$} & \multicolumn{1}{l}{$\begin{array}{l}\text { Action plan findings and definitions of } \\
\text { measures as reported in original papers }\end{array}$} \\
\hline Romer ${ }^{30}$ (2005) & $\begin{array}{l}\text { Any written asthma } \\
\text { management plan - no } \\
\text { details given. }\end{array}$ & $\begin{array}{l}\text { Quality improvement } \\
\text { learning collaborative for } \\
\text { general practice teams } \\
\text { (doctor, nurse \& } \\
\text { administrative assistant). } \\
\text { Participants had training, } \\
\text { coaching \& performance } \\
\text { feedback. Not stated } \\
\text { who provided training or } \\
\text { coaching. }\end{array}$ & $\begin{array}{l}\text { No significant differences between intervention \& control } \\
\text { groups in the proportion of participants receiving an AP at } \\
12 \text { months. } \\
\text { Duration: } 12 \text { months. } \\
\text { month study period compared to 41\% of the control group. } \\
\text { study period was greater amongst the control group (4\%) } \\
\text { than intervention group (1\%). The intervention was } \\
\text { therefore found to have no overall effect. } \\
\text { Definition of AP measure: any written AP reported by a } \\
\text { patient as having been received in the last year. }\end{array}$ \\
\hline
\end{tabular}

with subsequent educational reinforcement $(p=0.008)$. However, all educational studies except Osman' ${ }^{25}$ were ' $C$ 'graded, thus weakening the strength of this evidence.

Two Australian studies ${ }^{22,29}$ used telephone consultations to reinforce action plan use. Whilst Adams ${ }^{22}$ provided monthly telephone calls over a year from a non-healthcare worker, Khan $^{29}$ offered one-off telephone calls from asthma educators. Khan ${ }^{29}$ reported a significant increase in people with asthma having action plans $(p=0.001)$, and both studies reported increases in other measures including understanding and use. Kemple, ${ }^{27}$ in a 'B'-graded UK study, sent postal prompts to adults with asthma in their general practice inviting them for review enclosing partially personalised action plans for completion during their consultation. Participants receiving the intervention reported greater understanding of how to use their plans $(p=0.05)$ and higher rates of action plan usefulness $(p=0.21)$ than the control group or participants receiving blank action plans. ${ }^{27}$

Two studies used asthma clinics to promote action plan use. ${ }^{20,26}$ Conducted in different countries and settings, both studies reported increased ownership at six months postintervention ${ }^{20,26}$ although only Salisbury ${ }^{26}$ reported a statistically significant increase $(p=0.001)$.

Two further studies examined the effectiveness of asthma management systems. ${ }^{28,31}$ Glasgow's 'A'-graded trial' ${ }^{28}$ studied the efficacy of the comprehensive ' $3+$ plan'. This intervention consists of self-management training, asthma care and action plan development, combined with an active recall system inviting children with asthma for general practice review. In this study, more children receiving the intervention had action plans (44\%) compared to those receiving usual general practitioner (GP) care (34\%)(OR 2.2 [95\% Cl 1.2-4.1]). ${ }^{28}$ This means that five children (95\% Cl 3-41 children) would need to receive this intervention for one extra action plan to be given.

Rasmussen $^{31}$ also used an asthma management system consisting of an Internet-based tool with physician monitoring in Denmark. In this ' $C^{\prime}$-graded study, participants using the Internet system reported significantly higher action plan use $(p<0.001)$ at six months post-intervention than those receiving specialist out-patient or GP monitoring. ${ }^{31}$

Interventions aimed at health professionals

Clark ${ }^{21}$ provided evidence of medical education (based on the theory of self-regulation) encouraging action plan use. This study suggests that such education may facilitate action plan use for up to two years post-intervention; for example, more doctors $(p=0.02)$ who received the education commended parents of children with asthma for taking the right management action. However, this study was quality graded ' $C$ '. ${ }^{21}$

Homer $^{30}$ conducted a practice-based quality improvement and educational intervention. However, this showed no overall effect on action plan ownership, possibly due to contamination between study groups. ${ }^{30}$

\section{Primary care perspectives}

Kemple $^{27}$ and Glasgow ${ }^{28}$ conducted their interventions in general practice. As the only ' $A$ '-graded study reviewed, Glasgow's study ${ }^{28}$ has the strongest implications for primary care practice although only children with asthma participated, whereas Kemple's ' $B^{\prime}$-graded study ${ }^{27}$ involved a wider range of adult participants (16-93 years). Heard's ${ }^{20}$ asthma clinic was practice-based; however, nursing input was from an asthma educator rather than a practice nurse and results were not statistically significant. Homer's practice-based quality improvement intervention ${ }^{30}$ showed no clinical effect.

None of the educational $\left.\right|^{18,19,21,23-25}$ or telephone prompting ${ }^{22,29}$ interventions focused specifically on general practice patients or practice-based health professionals, although four of these studies were conducted in community settings. 19,22,23,29

\section{Discussion}

The 14 trials in this review were of variable quality. Nonetheless, 
Table 2. Trial Participant and Recruitment Details.

\begin{tabular}{|c|c|c|c|c|c|}
\hline $\begin{array}{l}\text { Author } \\
\& \text { year }\end{array}$ & Country & $\begin{array}{l}\text { Age } \\
\text { (years) }\end{array}$ & $\begin{array}{l}\text { Gender } \\
\text { (Male/Female) }\end{array}$ & How recruited & $\begin{array}{l}\text { Numbers randomised } \\
\& \text { completed }\end{array}$ \\
\hline $\begin{array}{l}\text { Adams }^{22} \\
(2001)\end{array}$ & Australia & $\begin{array}{l}16-70 \\
\text { Mean: Peak Flow } \\
\text { (PF) group } 37.3 \text { years. } \\
\text { Symptom (SM) group: } \\
35.5 \text { years. }\end{array}$ & $\begin{array}{l}\text { PF group: } 40 \% / 60 \% \\
\text { SM group: } 38 \% / 62 \%\end{array}$ & $\begin{array}{l}\text { Via in \& out patient clinics at a } \\
\text { teaching hospital. }\end{array}$ & $134 / 134$ \\
\hline $\begin{array}{l}\operatorname{Cote}^{23} \\
(2001)\end{array}$ & Canada & $\begin{array}{l}\text { Over } 18 \\
\text { Mean (SD): Control } \\
\text { group: } 38 \text { (3); Limited } \\
\text { education (LE) group: } 35 \\
\text { (3); Structured education } \\
\text { (SE) group: } 34 \text { (2) }\end{array}$ & $\begin{array}{l}\text { Control group } \\
46 \% / 54 \% \\
\text { LE group } 33 \% / 67 \% \\
\text { SE group } 11 / 2 \text { (\% can } \\
\text { not be calculated due to } \\
\text { discrepancy in } \\
\text { reporting). }\end{array}$ & $\begin{array}{l}\text { Via emergency or out patient } \\
\text { clinics following acute asthma } \\
\text { exacerbation. }\end{array}$ & $\begin{array}{c}126 / 98 \\
\infty\end{array}$ \\
\hline $\begin{array}{l}\text { Garrett }^{19} \\
(1994)\end{array}$ & $\begin{array}{l}\text { New } \\
\text { Zealand }\end{array}$ & $\begin{array}{l}2-55 \\
\text { Intervention \& control } \\
\text { groups (respectively) } \\
2-5: 25 \% \text { v } 26 \% \\
\text { 6-14: } 19 \% \text { v } 21 \% \\
\text { 15-29: } 30 \% \text { v } 32 \% \\
30-55: 25 \% \text { v } 21 \%\end{array}$ & $\begin{array}{l}\text { Intervention \& control } \\
\text { groups (respectively) } \\
\text { Male: } 38 \% \& 46 \% \\
\text { Female: } 62 \% \& 54 \% \text {. }\end{array}$ & $\begin{array}{l}\text { After attending emergency } \\
\text { room for acute asthma. }\end{array}$ & $500 / 451$ \\
\hline $\begin{array}{l}\text { Heard }^{20} \\
(1999)\end{array}$ & Australia & $\begin{array}{l}\text { 5-64 } \\
\text { Mean age: } \\
\text { Intervention: } 27.5 \\
\text { Control: } 26.3\end{array}$ & $\begin{array}{l}\text { Intervention: } 42 \% / 58 \% \\
\text { Control: } 45 \% / 55 \%\end{array}$ & $\begin{array}{l}\text { Patient participants had to } \\
\text { have attended a participating } \\
\text { general practice during the } \\
\text { study. }\end{array}$ & 195/191 \\
\hline $\begin{array}{l}\text { Homer }^{30} \\
(2005)\end{array}$ & $\begin{array}{l}\text { United } \\
\text { States }\end{array}$ & $\begin{array}{l}\text { Children: (median) } \\
\text { Intervention: } 8.5 \text { Control: } \\
9 \text { years. } \\
\text { Practice staff - age not } \\
\text { known. }\end{array}$ & $\begin{array}{l}\text { Intervention: } \\
64 \% / 36 \% \\
\text { Control group: } \\
\text { Male: } 60 \% / \text { Female } 40 \%\end{array}$ & $\begin{array}{l}\text { Paediatric practices within a } \\
\text { specified area volunteered } \\
\text { following presentations, } \\
\text { contacts with health networks } \\
\text { \& direct mailings. Children } \\
\text { were identified from practice } \\
\text { lists. }\end{array}$ & $\begin{array}{ll}\text { Practices: } & 43 / 40 \\
\text { Patients: } & 631 / 490\end{array}$ \\
\hline
\end{tabular}


$\mathrm{N}$ Ring et al.

\section{Table 2. Continued.}

\begin{tabular}{|c|c|c|c|c|c|}
\hline $\begin{array}{l}\text { Author } \\
\text { \& year }\end{array}$ & Country & $\begin{array}{l}\text { Age } \\
\text { (years) }\end{array}$ & $\begin{array}{l}\text { Gender } \\
\text { (Male/Female) }\end{array}$ & How recruited & $\begin{array}{l}\text { Numbers randomised } \\
\& \text { completed }\end{array}$ \\
\hline $\begin{array}{l}\text { Kemple }^{27} \\
(2003)\end{array}$ & UK & $\begin{array}{l}\text { Over 16s. Range 16-93 } \\
\text { Median } \\
\text { Group 1: } 38 \\
\text { Group 2: } 44 \\
\text { Group 3: } 43\end{array}$ & $\begin{array}{l}\text { Group 1: } 42 \% / 58 \% \\
\text { Group 2: } 40 \% / 60 \% \\
\text { Group 3: } 44 \% / 56 \%\end{array}$ & $\begin{array}{l}\text { Via general practice - all } \\
\text { asthmatic patients in a general } \\
\text { practice with a previous } \\
\text { prescription for inhaled } \\
\text { steroids. Practice identified as } \\
\text { a Research \& Development } \\
\text { practice. }\end{array}$ & $545 / 241$ \\
\hline $\begin{array}{l}\mathrm{Khan}^{29} \\
(2004)\end{array}$ & Australia & $\begin{array}{l}\text { Not stated - readers were } \\
\text { referred to a previous } \\
\text { paper [41] which states } \\
\text { participants were parents } \\
\text { of children aged 1-15 } \\
\text { years. }\end{array}$ & $\begin{array}{l}\text { Readers were referred } \\
\text { to previous paper [41] } \\
\text { which states gender } \\
\text { ratio (\% male/female) of } \\
\text { participating children } \\
\text { was } 65 / 35 \text {. }\end{array}$ & $\begin{array}{l}\text { Recruited following child's } \\
\text { discharge from hospital } \\
\text { emergency department. }\end{array}$ & $310 / 266$ \\
\hline $\begin{array}{l}\text { Osman }^{25} \\
(2002)\end{array}$ & UK & $\begin{array}{l}14-60 \\
\text { Median: } 29 \text { years. }\end{array}$ & $\begin{array}{l}\text { Total: } 34 \% / 66 \% \\
\text { Intervention group: } \\
40 \% / 60 \% \\
\text { Control group: } \\
28 \% / 72 \%\end{array}$ & $\begin{array}{l}\text { Following hospital admission } \\
\text { with acute asthma. }\end{array}$ & $280 / 271$ \\
\hline $\begin{array}{l}\text { Rasmussen }^{31} \\
\text { (2005) }\end{array}$ & Denmark & $\begin{array}{l}18-45 \\
\text { Internet group: } \\
28 \text { (18-44) } \\
\text { Specialist group: } \\
30 \text { (19-45) } \\
\text { GP group: } \\
30 \text { (20-45) }\end{array}$ & $\begin{array}{l}\text { Gender: \% Male/Female } \\
\text { Internet group: } \\
32 \%(n=27) / \\
68 \%(n=58) \\
\text { Specialist group: } \\
34 \%(n=30) / \\
66 \%(n=58) \\
\text { GP group: } \\
n=30 / n=58 \text { (unable to } \\
\text { calculate \% due to } \\
\text { reporting discrepancies). }\end{array}$ & $\begin{array}{l}\text { Participants were recruited } \\
\text { following distribution of an } \\
\text { asthma questionnaire. } \\
\text { Questionnaire recipients lived } \\
\text { in a specific hospital } \\
\text { catchment area. }\end{array}$ & $300 / 253$ \\
\hline $\begin{array}{l}\text { Salisbury }{ }^{26} \\
(2002)\end{array}$ & UK & Median age 13 years. & $\begin{array}{l}\text { Intervention group: } \\
51 \% / 49 \% \\
\text { Practice group: } \\
47 \% / 53 \% \\
\text { Control school: } \\
58 \% / 42 \%\end{array}$ & $\begin{array}{l}\text { From secondary schools } \\
\text { following completion of a } \\
\text { questionnaire. }\end{array}$ & $450 / 382$ \\
\hline $\begin{array}{l}\text { Yoon }^{18} \\
(1993)\end{array}$ & Australia & $\begin{array}{l}16-65 \\
\text { Mean: Intervention: } \\
30 \text { years. } \\
\text { Control: } 34 \text { years. }\end{array}$ & $\begin{array}{l}\text { Intervention/Control } \\
\text { Male } 24 \% / 28 \% \\
\text { Female } 76 \% / 72 \%\end{array}$ & $\begin{array}{l}\text { From hospital following severe } \\
\text { asthma exacerbation. }\end{array}$ & $76 / 56$ \\
\hline
\end{tabular}


from those studies demonstrating significant effects on action plan ownership and/or use, this review has identified some issues with important implications for healthcare practice and policy. Patient self-management education (groups or one-toone), school asthma clinics, one-off telephone prompting, and the 3+ asthma management system combined with active patient recall, increased action plan ownership. Patient selfmanagement education with six-month reinforcement, one-off telephone prompting, and Internet-based physician monitoring, increased action plan use. Medical education and postal prompts inviting adults to attend for asthma review combined with partially completed action plans were effective in facilitating action plan use - for example, doctors commending patients for taking the right asthma actions.

\section{Limitations and strengths of this review}

Action plans are recommended as good asthma care ${ }^{3,4}$ and are often included within complex self-management or guideline-based interventions. This made identification of relevant papers challenging because many studies reporting action plan data did not refer to an action plan (or equivalent term) in a paper's title or abstract. It is possible, therefore, that studies not included in this review exist. To minimise this potential limitation we adopted an over-inclusive approach to screening published papers and took extensive steps to identify unpublished studies.

Many studies were identified which included an action plan within their intervention but which did not report quantifiable action plan data. Whilst we actively attempted to contact lead authors for further information, project deadlines and the currency of author contact details publicly available constrained this process. To minimise this limitation, experts replying to requests for information during the literature searching process were sent a bibliography of studies meeting the review criteria and were asked to suggest any additional studies: no new studies were identified. Nevertheless, the authors acknowledge the possibility of publication bias, particularly given the relatively small number of studies identified as meeting our study criteria.

\section{Definitions and descriptions}

Although widely agreed definitions exist, ${ }^{1,4}$ the term 'action plan' was not used consistently within the literature; alternative terms such as 'self-treatment guidelines' were frequently used. This reflects the evolving understanding of asthma self-management, though consistent use of terminology would reduce confusion for people with asthma as well as practitioners. Inadequate descriptions of the action plans used is also frustrating to those attempting to replicate a study or implement findings, and highlights the need for researchers specifically to describe their action plans within publications. This review also highlights inconsistencies and inadequacies in the terms used to describe action plan outcomes. In future trials, if robust evidence for practice is to be developed, it is essential that researchers specifically define outcome measures such as 'use' and are consistent in their language when referring to such measures.

\section{Interventions, outcomes, and implications for practice}

Participants were most often recruited following contact with the hospital sector. 18,19,22-25,29 To avoid hospital re-admission, their motivation to self-manage and use action plans may have been higher, thereby increasing the effect of an intervention. These participants may also have exhibited different results from those with mild or stable asthma recruited from general practice. Lack of information in the original trials about the type of action plans used is another important issue since it is possible that the willingness of people with asthma to use their action plans may be influenced by their ability to individualise their plan to their circumstances.

Few studies in this review provided data for asthma subgroups - specifically patients of different genders, ethnicities, socio-economic profile, and older age patients. So, it is not possible to understand fully which interventions within this review will work best in promoting action plan use amongst different groups of patients. The absence of such data is particularly important for those from ethnic minority groups who have poorer asthma outcomes, and supports an earlier call for trial interventions to be evaluated in such populations. ${ }^{34}$ The use of pictorial action plans in future interventions might enable wider trial participation of those who have previously been excluded from trials - for example, those lacking English literacy or with mild learning disabilities.

Some interventions used nurses with specialist asthma training. ${ }^{20,24-26}$ Yet, not all clinical nurses providing asthma care, particularly in general practice, have received specialist training, ${ }^{12}$ so the effectiveness of an intervention may be greater if trial research nurses (with specialist training) are used rather than clinical nurses (with no such training). Research interventions may also have extra resources not otherwise available (including staff time and equipment); for example, participants in one study ${ }^{18}$ were lent peak flow meters if they could not afford to buy one, which may have influenced study outcomes.

Nonetheless, the reviewed trials suggest ways in which health professionals can promote effectively the use of action plans. However, for patients to benefit, application to everyday practice is crucial. Some interventions have greater potential for practical application than others. For example, interventions used by Kemple ${ }^{27}$ and Glasgow ${ }^{28}$ may be more easily integrated into general practice within the context of annual asthma reviews than Adams' monthly telephone calls over a year from a non-healthcare worker. ${ }^{22}$ The four 'A/B' quality studies $25,27,28,30$ are especially important as these are likely to yield particularly 
valid findings. Unfortunately, Homer's quality improvement intervention $^{30}$ cannot be recommended for practice as it showed no overall effect; but as the only study with doctor, nurse and administrative participants it supports the need for future interventions focusing on multi-disciplinary teams.

Glasgow's study which demonstrated that proactive asthma management increased action plan ownership ${ }^{28}$ is especially important as the only ' $A$ '-graded trial. Patient education, establishing individual action plans, and regular follow up, are key elements of an asthma management programme. ${ }^{4}$ Glasgow's study addressed these elements through the $3+$ asthma management plan combined with an active patient recall system. ${ }^{28}$ The Australian $3+$ plan provides financial incentives for general practices if they demonstrate that patients with moderate-to-severe asthma have regular asthma-related consultations, including medication review, patient education and action plan provision, over a specific period. ${ }^{35}$ Although the value of rewarding organised asthma care through this system has been recognised, ${ }^{36}$ there have been barriers to its implementation. Not all Australian GPs use the $3+$ plan, citing administrative workload ${ }^{35}$ and the need for flexibility in different practice situations ${ }^{36}$ as barriers to its use. This suggests that whilst the Glasgow intervention can increase action plan ownership, ${ }^{28}$ further consideration needs to be given as to how the key elements can be incorporated effectively into routine care.

Two 'B'-graded studies are also clinically relevant; Osman's study which demonstrated increased action plan ownership following asthma nurse education pre-hospital discharge, ${ }^{25}$ and Kemple's study which used postal prompts, invitations for asthma review and partially completed action plans to improve patient understanding of their use. ${ }^{27}$ The potential significance of the Osman, ${ }^{25}$ Kemple, $^{27}$ and Glasgow ${ }^{28}$ studies to clinical practice is also increased because these interventions were conducted in established healthcare systems rather than clinical settings contrived for research purposes.

\section{Implications for primary care practice}

Overall, this review highlights the lack of robust evidence for GPs and practice nurses on the best and most practical ways to sustain action plan use amongst their patients over the longer term. Studies by Kemple ${ }^{27}$ and Glasgow ${ }^{28}$ strengthen the importance of the annual asthma review as an opportunity for distributing and reinforcing action plan use routinely. Kemple's study also indicates that action plan use can be facilitated by sending patients a personalised partiallycompleted action plan which can be subsequently completed during the patient's asthma review. ${ }^{27}$ Primary care teams wanting to promote action plan use amongst their patients should therefore consider integrating action plan distribution and reinforcement into existing organisational systems. Within the UK, consideration could also be given to linking action plan provision to the Quality and Outcomes Framework asthma criteria. ${ }^{37}$

\section{Future research}

Patient self-management education, reinforcement and prompting, school asthma clinics, and asthma management systems, all increase patient ownership or initial use of action plans up to one year post-intervention, but more research is required to determine sustainability. As studies were more likely to report action plan ownership rather than use, researchers should concentrate on determining the effect of specific interventions on action plan usage, especially over the longterm and amongst asthma sub-groups. Crucially, outcome measures such as action plan use need to be specifically defined in future studies. Within the UK a research priority would be to develop and trial a multi-disciplinary general practice-based intervention aimed at initiating and sustaining action plan use within the context of the national Quality and Outcomes Framework. ${ }^{37}$ The use of educational interventions with primary care patients and/or professionals also requires further investigation. Finally, as most studies reviewed were judged to have a high potential for bias, methodological quality of future trials in this area should be improved.

\section{Conclusions}

Effective promotion of action plans requires interventions to initiate and sustain their use by health professionals and people with asthma. This review highlights several possible ways to initiate action plan use through increasing distribution and ownership of action plans by clinical practitioners in different sectors - for example, during pre-hospital discharge patient education ${ }^{25}$ and within school asthma clinics. ${ }^{26}$ Two of the highest quality papers also demonstrate the effectiveness of interventions directed at the organisation of practice-based asthma care to increase ownership and patient understanding of action plans. ${ }^{27,28}$ However, this review highlights that whilst most people with asthma are managed in primary care there is a further need to evaluate how multi-disciplinary primary care teams can sustain action plan use in the long-term.

Gibson has previously noted that, in relation to action plan use, 'the problem is not what to do, but how to do it'. ${ }^{11}$ Our findings give some indication of how best to encourage the use of action plans within primary care, but also highlight the need for further research to understand fully how primary care practitioners can best maximise the use of action plans by those patients with asthma.

\section{Funding}

NHS Quality Improvement Scotland and Asthma UK (Scotland) funded this systematic review.

\section{Conflicts of interest}

AS co-chairs the Science and Research Committee of the British Thoracic Society. HP chairs the Self-management and Patient Education Evidence Review Group for the 
British Thoracic Society/Scottish Inter-collegiate Guideline Network asthma guideline.

\section{References}

1. Gibson P, Powell H, Coughlan J, Wilson A, Abramson M, Haywood P, et al. Selfmanagement education and regular practitioner review for adults with asthma (Cochrane Review). In: The Cochrane Library. Issue 3. Oxford: Update Software, 2002.

2. Asthma UK. The Asthma Charter. London: Asthma UK; 2004.

3. British Thoracic Society \& Scottish Inter-collegiate Guideline Network. British guideline on the management of asthma: quick reference guide. Edinburgh: BTS \& SIGN; 2004.

4. Global Initiative for Asthma (GINA). Pocket Guide for Asthma Management and Prevention: a pocket guide for physicians and nurses. Updated from the NHLBIMHO Workshop Report (Publication 02-3659). Bethesda (Maryland): National Institutes of Health, National Heart, Lung and Blood Institute; 2005.

5. Gibson PG, Powell H. Written action plans for asthma: an evidence-based review of the key components. Thorax 2004;59:94-9.

6. Department of Health. Chronic disease management and self-care. National Services Frameworks: a practical aid to implementation in primary care. London: Department of Health; 2002.

7. Department of Health \& Ageing. National Evaluation of the Sharing Health Care Demonstration Projects: Final Report - Executive Summary. Canberra: Australian Government; 2005.

8. Scottish Executive. Building a Health Service Fit for the Future: A National Framework for Service Change in the NHS. Edinburgh: Scottish Executive; 2005.

9. World Health Organisation. Preparing a healthcare workforce for the 21st century: the challenge of chronic conditions. Geneva: World Health Organisation; 2005.

10. National Institutes of Health. Self-management Fact Sheet. Bethesda (Maryland): National Institutes of Health, 2006.

11. Gibson P. Asthma action plans: use it or lose it. Prim Care Resp J 2004:13:1718. doi:10.1016/j.pcri.2003.12.001

12. Hoskins G, McCowan C, Donnan P, Friend J, Osman L. Results of a national asthma campaign survey of primary care in Scotland. Int J Qual Health Care 2005; 17:209-15

13. Asthma UK (Scotland). Be in Control materials: a survey of their use by health professionals in Scotland. Edinburgh: Asthma UK Scotland; 2006.

14. Gillies J, Sheikh A, Wiener-Ogilvie S, Huby G, Pinnock H, Partridge MR. Describing compliance with and identifying barriers and facilitators to the implementation of SIGN 63 (The British Guideline for the Management of Asthma) in a rural health board (Report No.: CZG/2/186). Edinburgh: Chief Scientist Office; 2006.

15. Sulaiman N, Barton C, Abramson M, et al. Factors associated with ownership and use of written asthma action plans in North-West Melbourne. Prim Care Resp J 2004; 13:211-17. doi:10.1016/j.pcrj.2004.04.002

16. Barton C, Sulaiman N, Clarke D, Abramson M. Caregiver's use of, and attitudes towards, written asthma action plans in North-West Melbourne. Prim Care Resp J 2005;14:143-6. doi:10.1016/j.pcri.2004.10.006

17. NHS Quality Improvement Scotland \& Asthma UK (Scotland) Promoting the use of Personal Asthma Action Plans in Scotland: project specification strand two. Edinburgh: NHS QIS \& Asthma UK (Scotland); 2005.

18. Yoon R, McKenzie D, Bauman A, Miles D. Controlled trial evaluation of an asthma education programme for adults. Thorax 1993;48:1110-15.

19. Garrett J, Mercer Fenwick J, Taylor G, Mitchell E, Stewart J, Rea H. Prospective controlled evaluation of the effect of a community based asthma education centre in a multi-racial working class neighbourhood. Thorax 1994;49:976-83.

20. Heard A, Richards I, Alpers J, Pilotto L, Smith B, Black J. Randomised controlled trial of general practice based asthma clinics. Med J Aust 1999;171:68-71.

21. Clark N, Gong M, Schork A, et al. Long-term effects of asthma education for physicians on patient satisfaction and use of health services. Eur Resp J 2000; 16:15-21.

22. Adams R, Boath K, Homan S, Campbell D, Ruffin R. A randomised trial of peakflow and symptom-based action plans in adults with moderate-to-severe asthma. Respirology 2001;6:297-304.

23. Cote J, Bowie D, Robichaud P, Parent J-G, Battisti L, Boulet L-P. Evaluation of two different educational interventions for adult patients consulting with acute asthma exacerbation. Am J Resp Crit Care Med 2001;163:1415-19.

24. Morice A, Wrench C. The role of the asthma nurse in treatment compliance and self management following hospital admission. Respir Med 2001;95:851-6.

25. Osman L, Calder C, Goddan D, et al. A randomised trial of self management planning for adult patients admitted to hospital with acute asthma. Thorax 2002; 57:869-74.

26. Salisbury C, Francis C, Rogers C, et al. A randomised controlled trial of clinics in secondary schools for adolescents with asthma. Br J Gen Pract 2002; 52:988-96.

27. Kemple T, Rogers C. A mailed personalised self-management plan improves attendance and increases patient's understanding of asthma. Prim Care Resp J 2003; 12:110-14.

28. Glasgow NJ, Ponsonby A-L, Yates R, Beilby J, Dugdale P. Proactive asthma care in childhood: general practice based randomised controlled trial. BMJ 2003; 327:659-65.

29. Khan M, O'Meara M, Stevermuer T, Henry R. Randomised controlled trial of asthma education after discharge from an emergency department. J Paediatr Child Health 2004;40:674-7.

30. Homer C, Forbes P, Horvitz L, Peterson L, Wypij D, Heinrich P. Impact of a quality improvement programme on care and outcomes for children with asthma. Arch Pediat Adolesc Med 2005;159:464-9.

31. Rasmussen L, Phanareth K, Nolte H, Backer V. Internet-based monitoring of asthma: a long term, randomised clinical study of 300 asthmatic subjects. J Allergy Clin Immunol 2005;115:1137-41.

32. Higgins J, Green S, Editors. Cochrane Handbook for Systematic Reviews of Interventions 4.2.5 [updated May 2005] Issue 3. Chichester: John Wiley \& Sons, Ltd.; 2005.

33. Medical Research Council. A framework for development and evaluation of RCTs for complex interventions to improve health. London: MRC; 2000.

34. Griffiths C. Respiratory disease: does ethnicity matter? Prim Care Resp J 2004; 13:63-4. doi:10.1016/j.prcj.2003.03.001

35. Zwar N, Comino E, Hasan I, Harris M. General practitioner views on barriers and facilitators to implementation of the Asthma 3+ Visit Plan. Med J Aust 2005; 183(2):64-7.

36. Beilby J, Zwar N, Burgess T, et al. Linking evidence to policy- lessons from the national evaluation of the Asthma 3+ Visit Plan in Australia. Prim Care Resp J 2006;15:185-214. doi:10.1016/j.prcj.2006.04.119

37. NHS Confederation \& British Medical Association. New GMS Contract 2003: investing in general practice. London: NHS Confederation \& BMA; 2007.

38. Clark N, Gong M, Schork A, et al. Impact of education for physicians on patient outcomes. Pediatrics 1998;101:831-6.

39. Moher D, Cook DJ, Eastwood S, Olkin I, Rennie D, Stroup D. Improving the quality of reports of meta-analyses of randomised controlled trials: the QUORUM statement. Lancet 1999;354:1896-900.

40. Woolcock A, Rubinfeld A, Seale J, Landau L, Antic R, Mitchell C. Asthma management plan. Med J Aust 1989; 151:650-3.

41. Khan M, O'Meara M, Henry R. Background severity of asthma in children discharged from the emergency department. J Paediatr Child Health 2003; 39:432-5.

\section{Available online at http://ww w.thepcrj.org}

